\title{
Molecular Engineering of Crystalline Nano-optomechanical Transducers
}

Jacqueline M. Cole ${ }^{1,2,3,4,{ }^{*}}$

${ }^{1}$ Cavendish Laboratory, University of Cambridge, J. J. Thomson Avenue, Cambridge, CB3 OHE, UK.

2 ISIS Neutron and Muon Facility, STFC Rutherford Appleton Laboratory, Harwell Science and Innovation Campus, Didcot, OX11 0QX, UK.

${ }^{3}$ Department of Chemical Engineering and Biotechnology, University of Cambridge, West Cambridge Site, Philippa Fawcett Drive, Cambridge, CB3 OAS, UK.

${ }^{4}$ Argonne National Laboratory, 9700 S Cass Avenue, Argonne, IL 60439, USA.

* Email for correspondence: jmc61@cam.ac.uk

\begin{abstract}
Crystalline materials that behave as optical actuators and proceed via some form of nanooptomechanical mechanism are of particular interest for optical data storage[1] or quantum computing[2]. Nonetheless, the field is facing a dearth of suitable functional materials for applications. One possible material option is a series of compounds based on the generic formula, $\left[\mathrm{Ru}\left(\mathrm{SO}_{2}\right)\left(\mathrm{NH}_{3}\right)_{4} \mathrm{X}\right] \mathrm{Y}$, whose $\mathrm{SO}_{2}$ group manifests solid-state linkage photo-isomerization ( $\mathrm{X}$ is the trans-ligand to $\mathrm{SO}_{2} ; \mathrm{Y}$ is a counterion). This light-induced phenomenon causes these materials to act as photo-induced molecular switches [3-5] or molecular transducers [6,7] whose nanooptomechanical properties exist in the single-crystal state: a high-quality solid-state medium for single-photon control.

This talk will present the development of this family of materials towards such applications, via a range of advanced in situ 'photo-crystallography' and in-situ imaging experiments that capture the phenomenon in their light-induced state [8-10]. Results are enabling our understanding of the lightinduced molecular structure and nano-optomechanical properties of these light-induced solid-state actuators. Establishing this knowledge-base of structure-to-function relationships leads to the ultimate goal of being able to molecularly engineer these materials for a given device application.
\end{abstract}

\section{References}

[1] J. M. Cole, Zeit. fuer Krist. 223 (2008) 363.

[2] J. Boschmann, A. Veinsencher, D. D. Awschalom, A. N. Cleland, Nature Physics, 9 (2013) 712.

[3] J. M. Cole et al, Chem. Commun. (2006) 2448.

[4] A. E. Phillips, J. M. Cole, T. d'Almeida, K. S. Low, Phys. Rev. B 82 (2010) 155118.

[5] A. E. Phillips, J. M. Cole, T. d'Almeida, K. S. Low, Inorg Chem 51 (2012) 1204.

[6] S. O. Sylvester, J. M. Cole, Advanced Materials 25 (2013) 3324.

[7] S. O. Sylvester, J. M. Cole, P. G. Waddell, J. Am. Chem. Soc. 134 (2012) 11860.

[8] J. M. Cole, Chem. Soc. Rev. 33 (2004) 501.

[9] J. M. Cole, Acta Crystallogr. A64 (2008) 259.

[10] J. M. Cole, The Analyst, 136 (2011) 448. 